Original

\title{
Abuso de alcohol en el medio laboral, factores de riesgo para el consumo, e instrumentos de valoración aplicables en la vigilancia de la salud
}

\section{Alcohol abuse at workplace, risk factors and instruments for health surveillance}

\section{Danna C Ramírez Sánchez ', Katherin A Marínez Barroso ', Ángel Asúnsolo del Barco? \\ 1. Escuela Nacional de Medicina del Trabajo. Unidad docente I. Madrid. España. \\ 2. Universidad Alcalá de Henares. Facultad de Medicina. Departamento de Ciencias Sanitarias y Medico Sociales. Medicina Preventiva y Salud Pública. Alcalá de Henares (Madrid). España.}

Recibido: 06-09-11

Aceptado: 12-09-11

\section{Correspondencia}

Katherin A. Martínez Barroso

Embajadores 154, escalera externa, piso 2do A

28045 Madrid. España.

Tfno:680618465

e-mail: Katherinmbarroso@gmail.com

\section{Resumen}

Introducción: El consumo excesivo de alcohol es un problema de salud pública, según la OMS anualmente mueren 2,5 millones de personas por esta causa, el sector laboral no escapa a esta situación, creando la necesidad detectar precozmente el consumo de alcohol en los trabajadores.

Objetivos: Identificar los factores de riesgo para el consumo de alcohol en el medio laboral, y describir instrumentos útiles para el médico del trabajo, en el reconocimiento de trabajadores con problemas de abuso de alcohol.

Método: Se consultaron distintas bases de datos PUBMED, CISDOC, SCIELO, LILACS, MEDLINE The cocrhane library. Se evaluaron resúmenes y se recuperaron a texto completo aquellos relacionados con abuso de alcohol en población trabajadora.

Resultados: Se encontraron estudios epidemiológicos, de casos y controles, publicaciones de organismos oficiales y revistas de salud laboral. Estos evaluaron factores psicosociales y alcohol, algunos estudios usaron medidas de cribaje como cuestionarios AUDIT, CAGE y MALT para diagnosticar consumo perjudicial de alcohol.

Conclusiones: Se identificaron factores de riesgo en la población trabajadora, relacionados con el ambiente laboral, organización del trabajo, cualificación profesional y factores individuales: edad, sexo, y nivel socio-cultural. El uso de cuestionarios para detectar consumo de alcohol fue considerado la mejor opción, por fácil aplicación y bajo coste. Es necesario promover el uso de herramientas aplicables en el reconocimiento y prevención del abuso de alcohol en la población trabajadora.

Med Segur Trab (Internet) 2011; 57 (224) 190-209

Palabras clave: Abuso de alcobol, medio laboral, lugar de trabajo, factores de riesgo, CAGE, AUDIT. 
Abstract

Background: The alcohol abuse (AA) is a public health problem that causes 2.5 million deaths annually (WHO), the labor sector is not immune to this situation, creating the need for early detection of AA on workers.

Objective: To identify risk factors and instruments for health surveillance of workers with AA problems.

Methods: We reviewed the literature, PUBMED, CIS-DOC, SCIELO, LILACS, MEDLINE and the Cochrane Library. We included studies relating to AA in the working population, risk factors and consequences in the labor sector. They were classified according to level of evidence (Scottish Intercollegiate Guidelines Network, SIGN).

Results: There were 75 articles, 31 met the criteria for inclusion: A 2+ evidence study. Fifteen evidence 3 and sixteen with evidence level 4. Alcohol was the most consumed psychoactive substance in the working population prevalence of $77.8 \%$ in men and $72.9 \%$ in women. It was considered a risk factor for the AA, nonqualified. Two studies with evidence level 4 reported the increased accident rates and absenteeism related to AA. The AA screening methods used were CAGE and AUDIT .

Conclusions: The risk factors were identified in the working population, related to work organization, skill levels and individual factors. The use of CAGE and AUDIT for detection of AA was considered the best option for easy application and low cost. Insufficient documentation was found on the effects of AA, suggesting the promotion of study of these issues and assess the use of screening measures to enhance prevention.

Med Segur Trab (Internet) 2011; 57 (224) 190-209

Key words: Alcohol abuse, labor sector, risk factors, workplace, CAGE, AUDIT. 


\section{INTRODUCCIÓN}

El consumo de alcohol de forma nociva constituye hoy en día un grave problema de salud pública. Según un reciente informe de la Organización Mundial de Salud (OMS) de 2010, anualmente el consumo excesivo de alcohol causa la muerte de 2,5 millones de individuos, ocupando a nivel mundial el octavo lugar entre los factores de riesgo de muerte ${ }^{1}$.

El sector laboral, no escapa de esta realidad, y por esta razón las políticas de la Organización Internacional del Trabajo (OIT) han cambiado en los últimos 10 años, orientándose a prevenir el uso nocivo de alcohol y otras drogas en el lugar de trabajo, y definiendo esta situación como cualquier otro problema de índole sanitario ${ }^{2}$.

En España existe una prevalencia elevada de consumo de alcohol tanto en la población general como en la trabajadora ${ }^{3}$. Una de las principales explicaciones es que las bebidas alcohólicas son sustancias de fácil acceso, aceptadas por la sociedad, y que tienen una gran tradición cultural ${ }^{4}$. Según el informe de la Encuesta Domiciliaria sobre Alcohol y Drogas en España EDADES 2009-2010, el 78,7\% de la población entre 15-64 años ha consumido bebidas alcohólicas alguna vez en el último año, el 63,3\% en el último mes, y un $11 \%$ lo ha consumido todos los días durante el último mes ${ }^{5}$. En 2007-2008 se realizo una encuesta de consumo sustancias psicoactivas en el ámbito laboral, en España y el alcohol como en años anteriores, resultó ser la sustancia más consumida por la población laboral ${ }^{6}$.

Este consumo perjudicial de bebidas alcohólicas se traduce en alteración de la salud de los trabajadores, del rendimiento laboral, productividad de las empresas, incumplimiento de la seguridad en la práctica laboral y genera costes económicos para el empresario y los sistemas de salud pública ${ }^{7}$.

Distintas poblaciones laborales han sido analizadas a razón de la problemática que representa el consumo perjudicial de alcohol y sus consecuencias, y una gran parte de estas investigaciones se han apoyado en medidas directas de cribaje usando cuestionarios como el CAGE (del acrónimo inglés Cutting down, Annoyance by criticism, Guilty feeling y Eye openers) y el AUDIT ( Alcohol Use Disorders Identification Test) entre otros, ya que la entrevista clínica dirigida a reconocer estos problemas resulta muy compleja y extensa, y los marcadores biológicos respectivos de laboratorio tienen limitaciones propias por su recolección y $\operatorname{coste}^{8}$, de hecho algunos autores aseguran que cuestionarios cortos como el CAGE parecen hacer buena correlación con el diagnóstico clínico de alcoholismo y por su alta sensibilidad en casos graves puede ser mejor predictor de dependencia de alcohol que algunos marcadores bioquímicos ${ }^{9}$. Estas cualidades acerca de los instrumentos de valoración de problemas relacionados con alcohol toman vital importancia a la hora de examinar a una población tan peculiar y especial como lo es la población trabajadora.

Por estas razones detectar de forma precoz el consumo excesivo de alcohol resulta un reto para la Salud Ocupacional, que crea la necesidad de buscar herramientas ${ }^{4}$ que permitan al médico de Trabajo identificar a estos trabajadores, seguirles y de ser necesario incluirles en programas de desintoxicación, con el objetivo fundamental de prevenir o disminuir factores de riesgo y efectos perjudiciales relacionados con el consumo de alcohol en el lugar de trabajo.

\section{OBJETIVO}

Identificar los factores de riesgo para el consumo excesivo de alcohol en el medio labora, y describir instrumentos útiles para el médico del trabajo, en el reconocimiento y seguimiento de trabajadores con problemas de abuso de alcohol aplicables en la vigilancia de la salud. 


\section{DEFINICIÓN DE TÉRMINOS}

- Unidad de Bebida Estándar UBE: Equivale a 10 gramos de alcohol puro.

- Consumo de bajo riesgo: Se consideran límites de bajo riesgo los inferiores a 17 UBE por semana para hombres y 11 UBE para mujeres.

- Consumo peligroso: Se considera entre 17-28 UBE en hombres y 11-17 UBE en mujeres.

- Consumo de riesgo o perjudicial: Se considera >28 UBE por semana en hombres y $>17 \mathrm{UBE}$ en mujeres. También se considera consumo de riesgo tomar 5 UBE en una sola ocasión. El consumo de riesgo se puede considerar en caso de antecedentes familiares de alcoholismo aunque el consumo sea bajo.

- Abstemio: Persona que no consume alcohol de manera habitual, aunque ocasionalmente consuma una pequeña cantidad.

- Bebedor con problemas o patrón de consumo desadaptativo: Individuo que como consecuencia del consumo de alcohol ha tenido o presenta alguna alteración en su salud, y/ó algún problema de orden familiar, laboral, legal o económico.

- Trastornos relacionados con el alcobol (TRA): Individuo con cualquier deterioro del estado físico (enfermedades hepáticas, neurológicas, digestivas entre otras) psíquico (enfermedades psiquiátricas) o social (accidentes, agresiones, pérdida de empleo, absentismo) en relación causal con su consumo de alcohol.

- Alcobolismo y Síndrome de dependencia de alcohol SDA: Aquel que cumple con los criterios diagnósticos de la Clasificación Internacional de Enfermedades CIE-10 o el Manual Diagnóstico o Estadístico de los trastornos mentales (DSM-IV) ${ }^{10}$.

\section{MÉTODOS}

Se revisaron las bases de datos PUBMED, CISDOC/International Laboral Organition (ILO), SCIELO, LILACS, MEDLINE, The cocrhane library, (número 1 2010). Se leyeron los resúmenes de cada estudio con la finalidad de determinar la relevancia y pertinencia del artículo y así considerar la recuperación del texto completo. Los autores evaluaron los estudios encontrados, se obtuvo el texto completo de 16 estudios. Cada autor evaluó de forma independiente 8 estudios. Finalmente se incluyeron en la revisión 12 artículos de todos los recuperados a texto completo. La estrategia de búsqueda se describe en el Anexo N. ${ }^{\circ} 1$.

La estrategia de búsqueda usada para cada base de datos fue la siguiente:

— PUBMED: (“alcoholism”[MESH Terms]) AND “workplace”[MESH Major Topic]

— ("Alcoholism" [MESH]) AND "occupational health" [MESH]

- CISDOC: "alcoholism and workplace".

- MEDLINE: Alcohol [Palabras del tlytulo] and Riesgo [Descriptor de asunto] and Trabajo [Palabras]

— LILACS: : Risk [Palabras del título] and alcohol consumption [Palabras] and laboral [Palabras]

Búsqueda de otros Recursos: Se realizó una búsqueda manual en las listas de referencias bibliográficas de los artículos seleccionados, encontrándose 4 artículos adicionalmente. Se obtuvieron publicaciones de organismos oficiales de salud ocupacional OIT, Ministerio de Salud Política Social e Igualdad, OMS, publicaciones de revistas de Medicina Ocupacional, Salud y Seguridad en el Trabajo, y libros de psiquiatría, obteniendo en total 15 publicaciones.

Se establecieron como criterios de selección: Artículos de estudios relacionados con ámbito laboral, que aportaran datos epidemiológicos, factores de riesgo, métodos diagnósticos, métodos de cribado (Cuestionario CAGE, AUDIT, MALT) y prevención de consumo de alcohol. 
Se incluyeron los artículos cuyo resumen tratará los siguientes aspectos: alcoholismo, población trabajadora, factores de riesgo para el consumo de alcohol dentro y fuera del trabajo, escalas y medidas de valoración de consumo excesivo de alcohol. Se excluyeron los artículos cuyo resumen desarrollara aspectos de detección de alcoholismo usando otras herramientas distintas a los cuestionarios, como marcadores biológicos, y artículos cuyo resumen exploraba las consecuencias y efectos negativos del consumo de alcohol relacionadas con el deterioro de la salud, aspectos psiquiátricos y terapias de desintoxicación.

\section{RESULTADOS}

En el periodo de revisión bibliográfica se encontraron 31 referencias con la siguiente distribución:

Estudios Epidemiológicos: se hallaron 13, de los cuales algunos se enfocaron en describir los factores de riesgo psicosocial y los derivados del ambiente y tipo de trabajo que podían aumentar el riesgo de consumo perjudicial de alcohol así como distinguir determinados sectores laborales y diferencias entre los turnos de la jornada donde se evidenciaba mayor uso de bebidas alcohólicas, un total de 9 artículos relacionaron estos factores de riesgo y la aplicación cuestionarios CAGE, AUDIT y MALT. En 3 estudios se centraron en la validación de los cuestionarios más comúnmente usados para el cribaje del consumo excesivo de alcohol. Por ultimo una revisión bibliográfica se oriento a relacionar el consumo de alcohol y accidentes laborales en España.

Estudios Analíticos; uno de casos y controles en el que se observó la disminución del consumo perjudicial de alcohol posterior a realizar una intervención formativa sobre las consecuencias del consumo excesivo en los trabajadores que resultaron con alto riesgo de alcoholismo al ser evaluados, y 2 estudio de cohorte prospectivo que evaluaron factores de riesgo psicosocial y ambiente de trabajo, y accidentalidad y consumo de alcohol Finalmente se recopilaron 15 publicaciones de distintas revistas (Salud $y$ Seguridad en el Trabajo, Salud y Drogas, Trastornos adictivos) y organismos oficiales de relevancia en el ámbito de salud laboral, así como bibliografía de psiquiatría.

\section{FACTORES DE RIESGO PARA EL CONSUMO DE ALCOHOL EN EL LUGAR DE TRABAJO}

Según los estudios evaluados se observó que dentro y fuera del ámbito laboral existen varios elementos que pueden contribuir a una dependencia de alcohol, el nivel de educación parece jugar un papel importante, en un estudio se pudo ver que los individuos que no habían terminado la escuela bebían más que los trabajadores con mayor grado de formación (54\% Vs 38\%), el inicio del consumo de alcohol se observó mayor en edades tempranas, y al aplicarles escalas de valoración de enfermedad común mental (GHQ) se observó que existía fuerte correlación entre consumidores de alcohol y alteración de la salud mental. Curiosamente en un estudio realizado en India la religión es uno factor de riesgo, los sujetos católicos mostraron 1,3 veces mayor riesgo de consumo de bebidas alcohólicas que los de religión Hindú ${ }^{11}$. La cultura y costumbres de cada región también influyen en el inicio del consumo de alcohol como forma de aceptación social, en la edad productiva se evidenció el aumento del consumo de alcohol probablemente influenciado por amigos, colaborando así al mantenimiento de la adicción ${ }^{12}$.

En un estudio transversal descriptivo (Brasil, Sao Pablo) cuya muestra estuvo representada por 100 trabajadores del colectivo que se dedica a la recolección de basura, teniendo como objetivo la descripción de los posibles factores responsables en el inicio de un problema relacionado con el alcohol y el impacto que esto traía para dicha población laboral, mediante el cuestionario: QRCAP (del inglés: Questionnaire Relation of alcohol consumption to the profession) y el AUDIT, mostró que la frecuencia de consumo de alcohol era elevada en este colectivo, donde el 94\% de los sujetos consumían 
alguna bebida alcohólica ocasionalmente, $15 \%$ de las mujeres resultaron consideradas alcohólicas, además la mayor parte de la los sujetos se mostraron preocupados de adquirir alguna enfermedad relacionada con el consumo perjudicial de alcohol, y esta gran parte refirió haber comenzado el consumo de alcohol en la adolescencia. Se observó que la falta de motivación psicosocial y baja autoestima derivada del desempeño de su trabajo les proporcionaba una significativa influencia para el consumo habitual de alcohol ${ }^{12}$.

Los trastornos afectivos como la depresión (posterior a una evaluación con la escala de depresión) se asocian fuertemente a los problemas relacionados con el alcohol, aunque también se hallaron asociaciones importantes con el descontento profesional por el desbalance esfuerzo-recompensa y la sensación de poco control sobre el trabajo haciendo difícil reconocer que situación se puede definir como precedente o predictor del consumo perjudicial de alcohol, según resultados obtenidos al estudiar una importante muestra trabajadora en tres países de Europa del Este ${ }^{13}$.

Las rotaciones de turno durante la jornada laboral se ha considerado en estudios anteriores posible factor de riesgo para presentar problemas relacionados con el alcohol, sin embargo en una investigación realizada a este tipo de colectivo con 990 sujetos, mostró que los trabajadores de doble turno presentaban una prevalencia menor de consumo elevado de alcohol, respecto a trabajadores de jornadas diurnas ${ }^{14}$.

Las diferencias de genero también han mostrado relevancia, en este sentido los hombres aparecen con mayor prevalencia de SDA, aunque se observó que en mujeres con trabajos que implicaban baja libertad de decisión se invertía esta proporción ${ }^{9,15}$. Resultados como este se observaron en un estudio realizado en Alemania en 1060 Veterinarios en el las mujeres tenian prevalencia de consumo de alcohol similar a los hombres e incluso las que se desmpeñaban en arreas prácticas a diferencia de los que trabajaban en consultas clínicas presentaban mas riesgo de consumo perjudicial de alcohol que los hombres ${ }^{16}$. Además otros estudios han evidenciado que las ocupaciones como la agricultura, la manufactura y las labores de construcción tienen mayor riesgo de iniciar problemas relacionados con el alcohol que otras ocupaciones ${ }^{9,15}$.

Otros estudios como el realizado en una provincia de Canadá en 2008 en una población 10,155 trabajadores se enfocó en evaluar riesgos de consumo de alcohol en el lugar de trabajo utilizando cuestionarios como el CAGE y relacionando estas respuestas con condiciones de trabajo; organización, nivel de exigencia de la tarea, grado de cualificación de los trabajadores, obteniendo como resultados una fuerte asociación entre la no cualificación de un trabajador y el aumento del riesgo de consumir alcohol OR:2,95, otro de los resultados indicaba un aumento del riesgo de consumir alcohol en trabajos penosos o poco reconocidos socialmente ${ }^{17}$.

La prevalencia de los problemas relacionados con el alcohol en la población laboralmente activa representa el principal objetivo de estudio para muchos investigadores, y relacionar esta prevalencia con factores psicosociales y propios del ambiente laboral aparece como punto diana para reconocer soluciones. Así en India se tomó en cuenta este argumento para estudiar a 1013 trabajadores de la industria, determinando a través del uso del test AUDIT y el GHQ12 (General Health Questionnaire, para el cribaje de enfermedad común mental) una prevalencia de $21,3 \%$ de la población total y una fuerte relación entre escalas altas de GHQ y el consumo perjudicial de alcohol, también se observó que $50 \%$ de los varones mostraron problemas con el consumo de alcohol como ya se ha mencionado en otros estudios, colaborando así a una valiosa base de investigaciones que clarifican los factores relacionados con esta problemática de salud ${ }^{11}$.

En España se calcula que entre el 5-25\% de la siniestrabilidad laboral está en estrecha relación con el consumo de alcohol en el lugar trabajo. Se estima que los trabajadores que consumen alcohol tienen 3 veces más accidentes en el trabajo que los abstemios $^{3}$. Los problemas detectados más frecuentemente han sido: absentismo, incremento de las bajas laborales, disminución del rendimiento, incumplimiento de la jornada laboral, malas relaciones en el ambiente de trabajo y conflictividad, propiciando 
un aumento de expedientes disciplinarios, despidos y en consecuencia aumento en las cifras de paro $^{18}$.

En 2007-2008 se realizo un estudio epidemiológico que se trataba de una encuesta sobre el consumo de sustancias psicoactivas en el medio laboral en una poblacion de 15,071 trabajadores, según los resultados obtenidos el alcohol era la sustancia psicoactiva más consumida por la poblacion trabajadora con una prevalencia en hombres y mujeres de $92.7 \%$ vs $87,6 \%$ respectivamente. El $12 \%$ de los hombres y el $4 \%$ de las mujeres presentaron consumo de riesgo y los factores psicosociales tambien jugaron un papel importante encontrandose asociación entre el consumo de alcohol y el bajo novel de ingresos, trabajar en el sector primario, construcción y hostelería (Ver anexos 2 y 3$)^{6}$.

Tabla 1. Estudios relacionados con los factores de riesgo para el consumo de alcohol

\begin{tabular}{|c|c|c|c|}
\hline Autor & Resumen & Año & Tipo de estudio \\
\hline $\begin{array}{l}\text { Hermansson } \\
\text { U, Helander A, } \\
\text { Brandt L, Huss } \\
\text { A, Rönnberg S. }\end{array}$ & $\begin{array}{l}\text { Deteccion } \\
\text { Riesgo consumo } \\
\text { de alcohol } \\
\text { medio laboral. } \\
\text { CAGE }\end{array}$ & 2010 & $\begin{array}{l}\text { Analitico } \\
\text { Casos y } \\
\text { Controles }\end{array}$ \\
\hline Marchand A, & $\begin{array}{l}\text { Abuso de } \\
\text { Alcohol trabajo } \\
\text { condiciones de } \\
\text { trabajo } \\
\text { CAGE }\end{array}$ & 2008 & $\begin{array}{l}\text { Descriptivo } \\
\text { Transversal } \\
\text { Muestra: } 10.055 \\
\text { Trabajadores }\end{array}$ \\
\hline
\end{tabular}

1) Aumento el $\mathrm{n}^{\mathrm{a}}$ de respuestas positivas en AUDIT al aumentar las horas de trabajo.

2) Tras comparar las respuestas de AUDIT antes y después de la intervención se presento una disminución de $53,3 \%$ a $22,8 \%$. respectivamente.

3) No se encontraron diferencias significativas entre la disminución de las respuestas positivas en el AUDIT y en las concentraciones de CDT tras la intervención.

1) Aumento el riesgo de consumir alcohol en tareas poco reconocidas socialmente ( collares azul) y en tareas que exige alta demanda de habilidades, destreza conocimiento (Collares blancos)

2) La mala organización del trabajo aumenta el riesgo de consumo de alcohol.

3) Se hallo fuerte asociación entre la no cualificación de un trabajador y el riesgo de consumir alcohol OR:2,95 en collares azul) y OR: 1,28 en collares blancos. La asociación no fue significativa con Profesionales Técnicos OR:0,97

Melanie Haring, Estrés consumo 2009 Descrptivo Petra strehmel, de alcohol Anja Schablon, Albert Nienhaus.

Mabuchi AS,

Factores

Oliveira DF, asociados al

Lima MP, consumo de

Conceicao MB, alcohol en

Fernandes H. el colectivo recolector de basura.

Transversal.
1) Las mujeres veterinarias en practica tienen mayor riesgo para el consumo de alcohol que los hombres veterinarios OR: 1,3

2) El $13 \%$ de los veterinarios dieron positivos en el test de CAGE de problemas relacionados con el alcohol (>2pts)

1) $66,7 \%$ de las mujeres consumían algún tipo de alcohol pero no fueron consideras dependientes

2) $52 \%$ del total de los sujetos no habían terminado educación básica

3) $94 \%$ de los sujetos consumen alguna bebida alcohólica ocasionalmente y la mayoría de estos individuos comenzaron el uso de alcohol durante la adolescencia

4) $15 \%$ de los sujetos fueron considerados alcohólicos 


\begin{tabular}{|c|c|c|c|c|}
\hline Autor & Resumen & Año & Tipo de estudio & Resultados \\
\hline $\begin{array}{l}\text { Ministerio } \\
\text { de Sanidad, } \\
\text { Politica Social } \\
\text { e Inmigracios }\end{array}$ & $\begin{array}{l}\text { Encuesta de } \\
\text { consumo de } \\
\text { sustancias } \\
\text { psicoactivas en } \\
\text { ambito laboral. }\end{array}$ & $\begin{array}{l}2007- \\
2008\end{array}$ & $\begin{array}{l}\text { Descrptivo } \\
\text { Trasnversal }\end{array}$ & $\begin{array}{l}\text { 1) El alcohol es la sustancia psicoactiva mas } \\
\text { consumida por la poblacion laboral. } \\
\text { 2) El } 12,2 \% \text { de los hombres y } 4,9 \% \text { de las muje- } \\
\text { res eran bebedores de alto riesgo. } \\
\text { 3) La prevalencia de consumo de alcohl es ma- } \\
\text { yor en hombres que en mujeres. } \\
\text { 4) Los trabajadores con de media jornada tpre- } \\
\text { sentan mayor consumo de alcohol. } \\
\text { 5) Los trabajadores con altos cargos directivos } \\
\text { tenian mayor prevalencia de consumo de al- } \\
\text { cohol de riesgo } \\
\text { 6) Los trabajadores con ocupaciones penosas } \\
\text { (frio, calor,malos olores) tienen mayor con- } \\
\text { sumo de bebidas alcoholicas. } \\
\text { 7) Los trabajadores de bajo nivel educativo } \\
\text { presentaron mayor consumo de riesgo de } \\
\text { alcohol. }\end{array}$ \\
\hline $\begin{array}{l}\text { M Bobak, H } \\
\text { Pikhart, R } \\
\text { Kubinova, S } \\
\text { Malyutina, } \\
\text { A Pajak, H } \\
\text { Sebakova, R }\end{array}$ & $\begin{array}{l}\text { Caracteristicas } \\
\text { psicosociales } \\
\text { en el trabajo y } \\
\text { su relación con } \\
\text { los problemas } \\
\text { relacionados }\end{array}$ & 2005 & $\begin{array}{l}\text { Descriptivo } \\
\text { Transversal }\end{array}$ & $\begin{array}{l}\text { 1) Encontraron asociación entre la ingesta ele- } \\
\text { vada de alcohol y los problemas relaciona- } \\
\text { dos con este consumo. } \\
\text { 2) Se observó que esta relación está en parte } \\
\text { mediada por sintomas depresivos de los su- } \\
\text { jetos estudiados. }\end{array}$ \\
\hline
\end{tabular}

Nikitin

$\begin{array}{ll}\text { Head, S A } & \text { Factores } \\ \text { Stansfeld, J } & \text { psicosocial } \\ \text { Siegrist } & \text { riesgo para SDA } \\ & \text { ambiente de } \\ & \text { trabajo }\end{array}$

2003 Prospectivo de cohorte trabajo

\begin{tabular}{|c|c|}
\hline $\begin{array}{l}\text { Hermansson } \\
\text { U, Knutsson A, } \\
\text { Brandt L, Huss } \\
\text { A, Ronnberg S, } \\
\text { Helander A. }\end{array}$ & $\begin{array}{l}\text { Alto riesgo } \\
\text { y consumo } \\
\text { perjudicial } \\
\text { de alcohol } \\
\text { trabajador de } \\
\text { jornadas diur } \\
\text { y por turnos }\end{array}$ \\
\hline $\begin{array}{l}\text { Gomez M.T, } \\
\text { Del Rio M. C, } \\
\text { Alvarez F. J. }\end{array}$ & $\begin{array}{l}\text { Alcohol y } \\
\text { Accidentes } \\
\text { laborales }\end{array}$ \\
\hline
\end{tabular}

2003 Descrptivo

Transversal
Revisión Bibliográfica
1) Se encontró relación entre la desproporción esfuerzo-recompensa y la dependencia de alcohol en estos trabajadores

2) El disbalance esfuerzo-recompensa resultó predictor de poca salud mental y problemas con las relaciones interpersonales.

3) En mujeres se encontró relación entre SDA y la poca libertad de decisión en el trabajo.

4) Se observó mayor prevalencia de SDA en las mujeres con alto grado profesional.

1) $20 \%$ de la muestra resultó positivo en el AUDIT y/o CDT

2) No se encontraron diferencias llamativas entre los resultados obtenidos en los trabajadores de jornada diurna y los de jornada por turnos.

3) Los trabajadores de doble turno mostró menor consumo de alcohol que jornada diurna.

Desde 1995-2001 se encontraron:

1) 13 estudios epidemiológicos: Unos describían la relación entre accidentalidad laboral bajas y consumo de alcohol. Y otros trataban del consumo de alcohol en el trabajo

2) 14 publicaciones variadas acerca del consumo de alcohol en el ámbito laboral. 


\begin{tabular}{|c|c|c|c|c|}
\hline Autor & Resumen & Año & Tipo de estudio & Resultados \\
\hline $\begin{array}{l}\text { Crang } \\
\text { Zwerling, } \\
\text { Nancy L } \\
\text { Sprince, Robert } \\
\text { B, Wallace, } \\
\text { Charles S. } \\
\text { Davis, Paul S. } \\
\text { Whiten, Steven } \\
\text { G. Heeringa. }\end{array}$ & $\begin{array}{l}\text { Alcohol y } \\
\text { lesiones } \\
\text { ocupacionales. }\end{array}$ & 1996 & $\begin{array}{l}\text { Analítico } \\
\text { De cohorte. }\end{array}$ & $\begin{array}{l}\text { 1) Se encontró mayor numero de lesiones ocu- } \\
\text { pacionales relacionadas con consumo de al- } \\
\text { cohol entre } 51-54 \text { años. } \\
\text { 2) Las lesiones disminuían al aumentar el nivel } \\
\text { de Educación. } \\
\text { 3) Según los resultados del Test de CAGE: } \\
\text {-A mayor numero de respuestas positivas } \\
\text { mayor riesgo de lesiones. } \\
\text {-En los trabajadores que consumían } 5 \text { o } \\
\text { mas copas al ida se encontró que tenían } \\
\text { 3-4 respuestas positivas en el test. } \\
\text {-Él numero de respuestas positivas dismi- } \\
\text { nuía a mayor nivel de Educación. Los tra- } \\
\text { bajadores de cuello azul ( trabajos peno- } \\
\text { sos) tomaban } 5^{\circ} \text { mas copas al ida y tenían } \\
\text { 3-4 respuestas positivas en el Test. }\end{array}$ \\
\hline
\end{tabular}

\section{MÉTODOS DE DETECCIÓN DE CONSUMO DE ALCOHOL}

Según la bibliografía consultada en el proceso de vigilancia médica en el ámbito laboral impera el uso de herramientas de cribaje que permitan reconocer el consumo perjudicial de alcohol en la población trabajadora y poder establecer soluciones oportunas e intensificar la prevención. Unas cuantas revisiones apoyan el uso de pruebas de cribaje en atención primaria ${ }^{19}$, y estas investigaciones han contribuido a que sean elegidas como herramientas útiles en el ámbito laboral para la detección de consumo de riesgo en trabajadores de distintos sectores.

Dentro de los instrumentos actualmente validados para la evaluación de problemas relacionados con el consumo de alcohol y dependencia alcohólica se encontraron como métodos aceptados y de mayor difusión los cuestionarios AUDIT, MALT y CAGE ${ }^{19,20}$.

El cuestionario CAGE desarrollado en 1970 por Edwing and Rouse, se trata de un test rápido que con cuatro preguntas de respuesta dicotómica (crítica social, sentimientos de culpa por el consumo de alcohol, ingesta matutina de alcohol, y necesidad de abandonar el consumo) ha demostrado ser un instrumento con aceptable consistencia interna $^{21} \mathrm{y}$ alta sensibilidad ${ }^{9}$, aunque otros investigadores no han considerado que tenga una adecuada sensibilidad, su uso se ha extendido en mayor medida gracias a su brevedad y facilidad de puntuación ${ }^{19}$.

Estas son las 4 preguntas que incluye el test:

1. ¿Ha tenido usted alguna vez la impresión de que debería beber menos?

2. ¿Le ha molestado alguna vez que la gente critique su forma de beber?

3. ¿Se ha sentido alguna vez mal o culpable por su costumbre de beber?

4. ¿Alguna vez lo primero que ha hecho por la mañana ha sido beber para calmar sus nervios o para librarse de una resaca? ${ }^{22}$. Se consideran que con 2 respuestas positivas existe elevada susceptibilidad de problemas de abuso de alcohol, mientras que 4 respuestas afirmativas son altamente indicativas de alcoholismo ${ }^{13}$.

El MALT (Munchner Alkoholismus Test) es un cuestionario muy utilizado en la actualidad para el diagnostico de dependencia alcohólica con la particularidad de ser muy útil en aquellos casos de dependencia encubierta ${ }^{11}$. Está compuesto con un mayor número de preguntas que el CAGE, constituido de dos partes, el MALT objetivo (MALT-O), consta de 7 ítems que son cumplimentados por el entrevistador y que recoge datos de la anamnesis, exploración física y laboratorio, y el MALT subjetivo (MALT-S), que es autocumplimentado por el paciente, incluye 27 ítems que exploran aspectos psicoconductuales y repercusiones del consumo de alcohol. (Ver anexo N. ${ }^{\circ}$ 4) $^{19,20}$. Para 
algunos autores este test ha demostrado ser menos sensible y de aplicación más complicada $^{8,23}$.

En la interpretación del cuestionario las respuestas positiva del MALT-0 suma 4 puntos, mientras cada respuesta positiva del MALT-S suma un punto. Se considera que puntuaciones iguales o superiores a 11 son diagnósticas de alcoholismo y entre 6 y 10 sospecha de alcoholismo o de alto riesgo de alcoholismo. Para algunos autores su sensibilidad es del $100 \%$ y su especificidad del $80 \%$ mientras que otros han citado una sensibilidad y especificidad de $92,7 \%$ y $90,9 \%$ respectivamente en los puntos de más alto rendimiento diagnostico del test ${ }^{19}$.

El AUDIT fue un proyecto desarrollado por la OMS en 1982, con la finalidad de detectar consumo de riesgo o perjudicial de forma precoz ${ }^{24}$. Particularmente fue creado para ayudar a los clínicos a identificar aquellas personas que podrían beneficiarse de la reducción o abandono del consumo de alcohol. Algunos investigadores le confieren una especificidad del $91-96 \%$ y una sensibilidad de 57-59\%, este cuestionario ha mostrado ser una herramienta útil en atención primaria, y de esta se destaca que en ocasiones puede estar limitado por la necesidad de invertir considerable tiempo para su aplicación ${ }^{25}$. El cuestionario consta de 10 preguntas acerca de las consecuencias de beber junto con preguntas sobre cantidad y frecuencia de consumo. La $1^{\text {era }}, 2^{\text {da }}$ y $3^{\text {era }}$ pregunta se refieren al consumo de riesgo de alcohol, las preguntas 4 a 6 están relacionadas con síntomas de dependencia y las preguntas 7 a 10 sobre consumo perjudicial de alcohol. Cada pregunta puntúa de 0 a 4, y la suma final permite identificar, en función del punto de corte, tanto los consumos de riesgo como la dependencia alcohólica (Ver anexo N. $\left.{ }^{0} 5\right)^{19,24}$.

El AUDIT según los estudios encontrados es uno de los cuestionarios mas usados en Estados Unidos, un estudio de casos y controles realizado en 990 empleados de una empresa de transporte evalúo a estos empleados tras cumplimentar el cuestionario AUDIT, y los resultados de un biomarcador en sangre (CDT) comparando las modificaciones de los parámetros antes y después de una intervención que consistía en formar a los trabajadores sobre el riesgo de consumir alcohol en el lugar de trabajo y las herramientas a las cuales podían acudir en caso de tener alguna adicción. Dos grupos recibieron la formación, un tercer grupo no la recibió, y los resultados que se obtuvieron dejaban claro que la intervención había disminuido el número de respuestas positivas en el cuestionario AUDIT del $51,2 \%$ al $22 \%$ los que se traducía en una reducción casi a la mitad de los bebedores de riesgo, y con al grupo que no recibió la formación no se encontraron diferencias en los resultados al evaluarlos en los 12 meses siguientes ${ }^{26}$.

En España el cuestionario más usado anteriormente era el CAGE, sin embargo nuevos estudios han incluido el AUDIT como es el caso del EDADES ${ }^{5}$ estudio que evaluó la prevalencia del consumo de alcohol y otras drogas en España en el periodo de 20092010 y que por primera vez introduce este cuestionario para la identificación de bebedores de riesgo. Otros estudios realizados en España han intentado relacionar el consumo de alcohol a través de los resultados de la aplicación simultanea de este cuestionario y pruebas biológicas en trabajadores del sector publico ${ }^{19}$.

El ISCA (Interrogatorio Sistematizado de Consumo de Alcohol) se trata de un cuestionario formado por 3 preguntas que incluyen Tipo de consumo, frecuencia, y cantidad. Se propone como punto de corte consumo $>28$ en hombres y $>17$ en mujeres para considerar consumo de riesgo ${ }^{24}$. Existen otros tantos cuestionarios para la detección de problemas relacionados con el alcohol, pero se debe resaltar la diferencia de utilidad que estos tienen respecto a los usados para determinar dependencias (medidas directas de dependencia), donde la prioridad es investigar el grado del problema y que probablemente serán menos usados por el médico del trabajo. Así los más frecuentemente utilizados para este fin son cuestionarios mas largos como el SADQ (Severity of Alcohol Dependence questionnaire) que hace hincapié en aspectos de la abstinencia y la ADS (Alcohol dependence scale) ${ }^{23}$.

Además el médico cuenta con las medidas y escalas de autoeficacia que ayudan a predecir la evolución del paciente alcohólico en términos de recaídas y plantearse 
objetivos en el tratamiento, determinando a través de cuestionarios como el "Cues for drinking questionnaire" la capacidad del individuo para abstenerse y controlar el consumo de alcohol ${ }^{23}$. Estos cuestionarios son usadas en mayor medida en psiquiatría, pero por tratarse de metodos de seguimiento se mencionan en este apartado.

Por último están los marcadores biológicos que a pesar de no ser objeto de este estudio, son usados también en el cribado y diagnóstico de consumo de alcohol, son de poca utilidad en muchos casos debido la variabilidad de los niveles en sangre en cada individuo en relación a conductas alimentarias, ejercicio físico, patologías asociadas entre otras, y por tanto su resultado positivo no confirma el diagnóstico pero podrían ser consideraoas una señal de alarma sobre el consumo de alcohol ${ }^{24}$. En países como Estados Unidos es legal realizar este tipo de pruebas a los trabajadores, incluso es obligada en algunos sectores laborales como el Sector Transporte ${ }^{27}$. A diferencia de España donde esto es considerado una violación a la intimidad del trabajador y acarrea una serie de conflictos éticos ${ }^{28}$.

Tabla 2. Estudios relacionados con los metodos de detección de consumo de alcohol

\begin{tabular}{|c|c|c|c|c|}
\hline Autor & Resumen & Año & Tipo de estudio & Resultados \\
\hline $\begin{array}{l}\text { Hermansson } \\
\text { U, Helander A, } \\
\text { Brandt L, Huss } \\
\text { A, Rönnberg S. }\end{array}$ & $\begin{array}{l}\text { Detección } \\
\text { Riesgo } \\
\text { consumo de } \\
\text { alcohol medio } \\
\text { laboral. } \\
\text { CAGE }\end{array}$ & 2010 & $\begin{array}{l}\text { Analítico } \\
\text { Casos y } \\
\text { Controles } \\
\text { Muestra: } 990 .\end{array}$ & $\begin{array}{l}\text { 1) Aumento el } \mathrm{n}^{\mathrm{a}} \text { de respuestas positivas en } \\
\text { AUDIT al aumenta las horas de trabajo. } \\
\text { 2) Tras comparar las respuestas de AUDIT an- } \\
\text { tes y después de la intervención se presento } \\
\text { una disminución de } 53,3 \% \text { a } 22,8 \% \text {. respec- } \\
\text { tivamente. }\end{array}$ \\
\hline Ramírez A. & $\begin{array}{l}\text { Detección } \\
\text { precoz } \\
\text { consumo de } \\
\text { alcohol AUDIT }\end{array}$ & 2010 & $\begin{array}{l}\text { Descriptivo } \\
\text { Transversal. } \\
\text { Muestra: }\end{array}$ & $\begin{array}{l}\text { 1) } 1 \% \text { son bebedores de bajo riesgo, } 7 \% \text { de alto } \\
\text { riesgo y el } 12 \% \text { abstemios. } \\
\text { 2) Se encontró débil correlación entre cuestio- } \\
\text { nario ISCA y AUDIT p }>0,01\end{array}$ \\
\hline $\begin{array}{l}\text { Pérula } \\
\text { DeTorres L, } \\
\text { Carmona- } \\
\text { Tamajón V, } \\
\text { Montero Pérez- } \\
\text { Barquero M, } \\
\text { Ruiz-Moral R, } \\
\text { Fernández- } \\
\text { García J, }\end{array}$ & $\begin{array}{l}\text { Diagnostico } \\
\text { alcoholismo } \\
\text { validación } \\
\text { prueba MALT }\end{array}$ & 2009 & $\begin{array}{l}\text { Descriptivo } \\
\text { Evaluación } \\
\text { de prueba } \\
\text { diagnostica. }\end{array}$ & $\begin{array}{l}\text { 1) El } 63 \% \text { de la población consumía alcohol; } \\
\text { mas en hombres que en mujeres } 94 \% \text { y } 45 \% \\
\text { respectivamente. } \\
\text { 2) El } 3,1 \% \text { cumplía criterios de abuso de alco- } \\
\text { hol . } \\
\text { 3) El punto de corte } 4 \text { del MALT-S obtuvo la } \\
\text { mejor combinación de sensibilidad } 92,6 \% \text { y } \\
\text { especificidad } 90,8 \% \text {. }\end{array}$ \\
\hline $\begin{array}{l}\text { Ministerio } \\
\text { de Sanidad } \\
\text { Política social } \\
\text { e Igualdad }\end{array}$ & $\begin{array}{l}\text { Encuesta } \\
\text { consumo } \\
\text { alcohol y } \\
\text { drogas } \\
\text { España }\end{array}$ & $\begin{array}{l}2009- \\
2010\end{array}$ & $\begin{array}{l}\text { Descriptivo. } \\
\text { Transversal } \\
\text { Muestra: } \\
20.109\end{array}$ & $\begin{array}{l}\text { 1) El alcohol es una sustancias mas consumi- } \\
\text { das con un } 79 \% \text {. } \\
\text { 2) Un } 78,2 \% \text { ha consumido alcohol en el ultimo } \\
\text { año, } 63,3 \% \text { ultimo mes, } 11 \% \text { a diario ultimo } \\
\text { mes. } \\
\text { 3) El cuestionario AUDIT mostró un } 7,4 \% \text { de la } \\
\text { población con consumo de riesgo y un } 0,3 \% \\
\text { muestra posible dependencia. }\end{array}$ \\
\hline $\begin{array}{l}\text { M Bobak, H } \\
\text { Pikhart, R } \\
\text { Kubinova, S } \\
\text { Malyutina, } \\
\text { A Pajak, H } \\
\text { Sebakova, R }\end{array}$ & $\begin{array}{l}\text { Caracteristicas } \\
\text { psicosociales } \\
\text { en el trabajo y } \\
\text { su relación con } \\
\text { los problemas } \\
\text { relacionados }\end{array}$ & 2005 & $\begin{array}{l}\text { Descriptivo } \\
\text { Transversal }\end{array}$ & $\begin{array}{l}\text { 1) Se encontró asociación entre la ingesta ele- } \\
\text { vada de alcohol y los problemas relaciona- } \\
\text { dos con este consumo. } \\
\text { 2) Se observó que esta relación está en parte } \\
\text { mediada por síntomas depresivos de los su- } \\
\text { jetos estudiados. }\end{array}$ \\
\hline
\end{tabular}




\begin{tabular}{|c|c|c|c|c|}
\hline Autor & Resumen & Año & Tipo de estudio & Resultados \\
\hline $\begin{array}{l}\text { Head, S A } \\
\text { Stansfeld, J } \\
\text { Siegrist }\end{array}$ & $\begin{array}{l}\text { Factores } \\
\text { psicosocial } \\
\text { riesgo para } \\
\text { SDA ambiente } \\
\text { de trabajo }\end{array}$ & 2003 & $\begin{array}{l}\text { Prospectivo de } \\
\text { cohorte }\end{array}$ & $\begin{array}{l}\text { 1) Se encontró relación entre la desproporción } \\
\text { esfuerzo-recompensa y la dependencia de } \\
\text { alcohol en estos trabajadores } \\
\text { 2) El disbalance esfuerzo-recompensa resultó } \\
\text { predictor de poca salud mental y problemas } \\
\text { con las relaciones interpersonales. }\end{array}$ \\
\hline $\begin{array}{l}\text { Clodag M. } \\
\text { Cashman, } \\
\text { Jani H. } \\
\text { Ruotsalainen, } \\
\text { Birgit A. } \\
\text { Reiner, Paul V. } \\
\text { Beirme, Jos H, } \\
\text { Verbeek }\end{array}$ & $\begin{array}{l}\text { Cribado de } \\
\text { Consumo de } \\
\text { Alcohol en } \\
\text { Conductores } \\
\text { profesionales } \\
\text { en la } \\
\text { prevención de } \\
\text { lesiones. }\end{array}$ & 2010 & $\begin{array}{l}\text { Descriptivo } \\
\text { Revisión } \\
\text { Bibliográfica. }\end{array}$ & $\begin{array}{l}\text { 1) La prueba de detección de drogas y alcohol } \\
\text { obligatoria al azar se asocio a un cambio in- } \\
\text { mediato en el numero de lesiones después } \\
\text { de la intervención. } \\
\text { 2) A largo plazo la prueba de detección presen- } \\
\text { to descenso de las cifras de lesiones }\end{array}$ \\
\hline $\begin{array}{l}\text { Campos-Arias } \\
\text { A, Barros- } \\
\text { Bermudez J. A, } \\
\text { Rueda-Jaimes G. }\end{array}$ & $\begin{array}{l}\text { Propiedades } \\
\text { piscométricas } \\
\text { del CAGE }\end{array}$ & 2009 & $\begin{array}{l}\text { Descriptivo } \\
\text { transversal. } \\
\text { Validación } \\
\text { de prueba } \\
\text { Diagnostica. }\end{array}$ & $\begin{array}{l}\text { El cuestionario CAGE demostró buena consis- } \\
\text { tencia interna. }\end{array}$ \\
\hline
\end{tabular}

\section{CONCLUSIONES}

La revisión de estos estudios ha permitido identificar factores de riesgo involucrados en el abuso de alcohol en la población trabajadora. Algunos de estos factores se ven claramente relacionados con el ambiente y organización del trabajo (tipo de profesión, carga laboral, funciones a desempeñar, factores derivados de estrés y presión en el lugar de trabajo, etc.), mientras que otros son propios de cada individuo y se pueden encontrar en la población general (edad, sexo, estado civil, nivel educativo y socio-cultural).

La legislación vigente en España en su Real Decreto: 1/1995 Estatuto de los trabajadores en el artículo 54.2.f considera como causa de despido el estado de embriaguez habitual o toxicomanía ${ }^{29}$. A pesar de existir evidente normativa al respecto, son muchos los trabajadores que consumen alcohol durante la jornada laboral, y muchos los accidentes laborales que no se han reportado.

Para evaluar el consumo perjudicial de alcohol se determinó que el uso de medidas de cribaje tipo cuestionario aparecen como la mejor opción para ejecutar durante la vigilancia de la salud y en consultas de atención primaria, por su fácil aplicación y bajo coste. Los cuestionarios AUDIT y CAGE mostraron niveles elevados ${ }^{23,25}$ de sensibilidad y especificidad, aunque se observó que para diagnosticar dependencia está indicado realizar estudios más extensos en aquellas personas donde exista alta sospecha o riesgo de alcoholismo.

Como parte clave de las consecuencias derivadas del abuso de alcohol se señaló en varias investigaciones el bajo rendimiento, la baja productividad y el aumento de accidentes laborales a causa de este problema en los trabajadores estudiados. Estas razones fundamentan la importancia en promover el uso de estas herramientas de valoración para evaluar la necesidad de implementar o reforzar medidas preventivas, o incluso de intervención, a fin de disminuir la prevalencia del consumo perjudicial de alcohol en la población trabajadora ${ }^{30,31}$.

Es importante destacar que a pesar de la utilidad de estas herramientas en la detección de consumo perjudicial de alcohol, se ha encontrado como limitación que estos cuestionarios evalua al trabajador de forma subjetiva, poniendo en duda la fiabilidad de sus resultados. 


\section{REFERENCIAS BIBLIOGRÁFICAS}

1. Organización Mundial de la Salud. Reducir el uso nocivo de alcohol una medida beneficiosa para la salud y para la comunidad. Ginebra: OMS; 2010.

2. Organización Internacional del Trabajo. Lanzamiento de una armada antisustancias.Progresos en la lucha contra alcohol y drogas en el trabajo. Rev. Trabajo num. 23; 1998.

3. Ochoa E, Madoz A. Consumo de alcohol y otras drogas en el medio laboral. Revista medicina y seguridad en el trabajo Madrid 2008, Vol. 54. 213.

4. Fernández J, Echeburúa E. El consumo excesivo de alcohol, un reto para la salud laboral. Revista Salud y Drogas 2001. año/vol 1 número 001. 17-39.

5. Ministerio de Sanidad política Social e Igualdad. Delegación del gobierno para el plan nacional sobre drogas Informe de la Encuesta domiciliaria de consumo de alcohol y drogas España. 2009/2010.

6. Ministerio de Sanidad Política Social e Igualdad. Observatorio Español sobre Drogas. Encuesta sobre consumo de sustancias psicoactivas en el ámbito laboral.España. 2007/2008

7. Gelder M, Mayou R, Geddes J. OXFORD PSIQUIATRIA. Edit. Marban 1999. 259-287

8. Wilson P, Spence S, Kvanagh D. Técnicas de entrevista clínica. Editorial Libergraf 1995. 167-187.

9. Head J, Stansfeld S A, Siegrist J. The psychosocial work enviroment and alcohol dependence: a prospective study. Occup Environ Med 2004 Vol. 61, 219-224.

10. Consumo de Alcohol. Guías sanitarias de prevención. Sociedad de Prevención de Asepeyo.2010.3-23.

11. Chagas M, Gaunekar G, Patel V, Kukalekar D., y Fernandes F. The prevalence and correlates of hazardous drinking in industrial workers: a study from Goa, India. Alcohol \& alcoholism 2003 Vol.38, Núm 1: 79-83.

12. Mabuchi AS, Oliveira DF, Lima MP, Conceicao MB, Fernandes H. The use of alcohol by workers of the garbage collection service. Revista latinoamericana Enfermagen 2007 Vol. 15 Núm 3: 446-52.

13. Babak M, Pikhart H, Kubinova R, Malyutina S, Pajak A, Sevakova H, Topor-Madry R, Mikitin Y, Caan W, Marmot M. The Association beetween psychosocial characteristics at work and problem drinking: A cross- sectional study of men in trhee Eastern European urban populations. Occup Environ Med 2005 Vol 62, 546-550.

14. Hermansson U, Knutsson A, Brandt L, Huss A, Ronnberg S, Helander A. Screening for high risk and elevated alcohol consumption in day and shift workers by use of the AUDIT and CDT. Occupational medicine, 2003 Vol. 53, 518-526.

15. Leggat P, Smith D. Alcohol related absenteeism: The need to analyse Consumption patterns in order to target screening and brief interventions in the workplace. Industrial Health 2009 Vol 47, 345-47.

16. Harling M, Strehmel P, Schablon A, Nienhaus A. Psychosocial stress, demoralization and the consumption of tobacco, alcohol and medical drugs by veterinarians. Journal of Occupational Medicine and Toxicology 2009, 4:4doi:10.1186/1745-6673-4-4

17. Marchand A. Alcohol use and misuse: what are the contributions of occupation and work organization conditions. C Public Health. 2008 Sep 24;8:333.

18. Gómez M, Javier F, Álvarez G. Alcohol y prevención de los problemas relacionados con el consumo de alcohol en el ámbito laboral. Revista Med. Seguridad en el Trabajo 2005, No 198: 65-72.

19. Ramírez Olmedo A. Identificación precoz de bebedores de riesgo en empleados públicos de la provincia de Cádiz. Revista Med Segur Trab 2010; 56 (219): 132-146.

20. Pérula de Torres LA, Carmona-Tamajón V, Montero M, Ruiz-Moral R, Fernández-García JA, MurielPalomino M. Validación del Münchner Alkoholismus Test Subjektiv para el diagnóstico de alcoholismo en población adulta. Revista de Atención Primaria.2009 Vol.41 Núm. 08. 0212-6567.

21. Campos-Arias A, J. Barros- Bermúdez J, Rueda-Jaimes G. Propiedades psicométricas del cuestionario CAGE para consumo abusivo de alcohol: Resultado de tres análisis. Revista Colombiana de Psiquiatría Abril- Junio 2009 Vol 38, Núm 2.

22. Bruguera M, Guai A, Salleras L, Robes J. Cribado del consumo excesivo de alcohol. Revista Medicina Clinica 1994; 102 supl 1:85-92.

23. Ewing JA. Detecting alcoholism. The CAGE questionnaire. JAMA 1984 Vol 252, Núm 14, $1905-1907$.

24. Rosón Hernández B. Consumo de riesgo y perjudicial de alcohol. Prevalencia y métodos de detección en la práctica clínica. Revista Galicia Clin 2008; 69 (1): 29-44. 
25. Álvarez S, Gallego P, Latorre C, Bermejo F. Revisión critica de la práctica clínica. Papel del test AUDIT (Alcohol Use Disorders Identification test) para la detección del consumo excesivo de alcohol. Medifan Octubre-Noviembre 2001. Vol. 11, Núm 9.

26. Hermansson U, Helander A, Brandt L, Huss A, Rönnberg S. Screening and brief intervention for risky alcohol consumption in the workplace: results of a 1-year randomized controlled study. PUBMED Alcohol Alcohol. 2010 May-Jun; 45(3):252-7.

27. Clogagh M, Ruotsalainen J, Greiner B, Beime P, Verbeek J. Cribado sobre consumo de alcohol y drogas entre conductores profesionales para la prevención de lesiones. 2010 num.1 ISSN 1745-9990.

28. Shahander B, Husbands, R. Estudio de casos: Consideraciones Eticas sobre el consumo de drogas y alcohol en el lugar de trabajo. Enciclopedia de Salud y Seguridad en el trabajo. 3era Edicion.2001. p19.25-19.29.

29. Boletin Oficial del Estado. Real Decreto 1/1995. Estatuto de los trabajadores.BOE num.75, 24/3/1995.

30. Crang Zwerling, Nancy L Sprince, Robert B, Wallace, Charles S. Davis, Paul S. Whiten, Steven G. Heeringa. Alcohol and ocupational injuries among older workers. Accid. Anal. And Prev. 1996. Vol.28, No. 3. 371-376.

31. Gómez M., del Rio M, Álvarez F. Alcohol y accidentes laborales en España: revisión bibliográfica, 19952001.Revista Trastornos Adictivos 2002.4(4):244-255. 


\section{ANEXO 1. ESTRATEGIA DE BÚSQUEDA EN BASES DE DATOS}

\begin{tabular}{|c|c|c|c|}
\hline Base de datos & Palabra clave & Resultados & $\begin{array}{l}\text { Artículos } \\
\text { seleccionados }\end{array}$ \\
\hline PUBMED & $\begin{array}{l}\text { Alcoholism (MesH Terms) and } \\
\text { workplace (MseH Major Topic) } \\
\text { Limites : Last } 10 \text { years }\end{array}$ & 17 & 3 \\
\hline PUBMED & $\begin{array}{l}\text { Alcoholism (Mesh) AND occupational health (Mesh) } \\
\text { Limits Last } 10 \text { years (PDat) } \\
\text { English and Spanish } \\
\text { Criterios de Selección: Artículos de estudios relacionados con } \\
\text { ámbito laboral, que incluyan datos epidemiológicos y/o facto- } \\
\text { res de riesgo, y/o métodos diagnostico ( Cuestionario CAGE, } \\
\text { AUDIT, MALT) y/o medidas preventivas y/o Legislación. }\end{array}$ & $\begin{array}{l}96 \\
36 \\
16\end{array}$ & 5 \\
\hline PUBMED & $\begin{array}{l}\text { "Alcohol Use Disorders Identification and Workplace” AUDI Test } \\
\text { Criterios de Selección; Artículos } \\
\text { De estudios relacionados con el método AUDIT aplicables al } \\
\text { ámbito laboral. }\end{array}$ & 9 & 1 \\
\hline CISDOC & $\begin{array}{l}\text { Alcoholism and Workplace } \\
\text { Limites: Idioma Español e Ingles. } \\
\text { Últimos } 10 \text { años } \\
\text { Criterios de Selección: Artículos de estudios relacionados con } \\
\text { ámbito laboral, que incluyan datos epidemiológicos y/o mé- } \\
\text { todos diagnósticos ( Cuestionario CAGE, AUDIT, MALT) y/o } \\
\text { medidas preventivas y/o Legislación relacionada. } \\
\text { Se descartaron artículos repetidos en otras búsquedas. }\end{array}$ & $\begin{array}{c}118 \\
30\end{array}$ & 3 \\
\hline SCIELO & $\begin{array}{l}\text { Alcohol ( Titulo) and } \\
\text { Trabajo (todos los índices) }\end{array}$ & 6 & 3 \\
\hline $\begin{array}{l}\text { The Cocrhane } \\
\text { Library }\end{array}$ & $\begin{array}{l}\text { Hazadous Drinking and } \\
\text { Ocupational Health }\end{array}$ & 2 & 1 \\
\hline LILACS & $\begin{array}{l}\text { Risk [Palabras del título] and alcohol consumption [Palabras] } \\
\text { and laboral [Palabras] }\end{array}$ & 5 & 0 \\
\hline MEDLINE & $\begin{array}{l}\text { Alcohol ( palabra del titulo) and } \\
\text { Riesgo (Descriptor de Asunto) } \\
\text { and trabajo (palabras) } \\
\text { Artículos repetidos en otras búsquedas }\end{array}$ & $\begin{array}{c}10 \\
2\end{array}$ & 0 \\
\hline
\end{tabular}




\section{ANEXO 2. FACTORES DE RIESGO PARA EL CONSUMO DE ALCOHOL}

\begin{tabular}{llcc}
\hline & & Hombres & Mujeres \\
\hline Edad & 16-34 Años & 5,0 & 2,6 \\
\multirow{2}{*}{ Nivel educativo } & 35-64 Años & 5,0 & 2,1 \\
& Sin estudios/estudios primarios & 8,7 & 3,3 \\
& Estudios secundarios & 4,5 & 2,2 \\
Estado civil & Estudios universitarios & 2,9 & 2,0 \\
& Soltero/a & 5,4 & 3,0 \\
& Casado/a - en pareja & 4,1 & 1,6 \\
País de nacimiento & 11,0 & 3,4 \\
& Separado/a - divorciado/a -viudo/a & 5,2 & 2,4 \\
Total & España & 4,1 & 2,2 \\
& Otros & $\mathbf{5 , 0}$ & $\mathbf{2 , 3}$ \\
\hline
\end{tabular}

Fuente: Encuesta Domiciliaria sobre Alcohol y Drogas en España. EDADES.2007.08. Observatorio Español sobre Drogas. 


\section{ANEXO 3. RIESGO DE CONSUMO DE ALCOHOL SEGÚN SECTOR LABORAL, CATEGORIA PROFESIONAL, Y JORNADA DE TRABAJO}

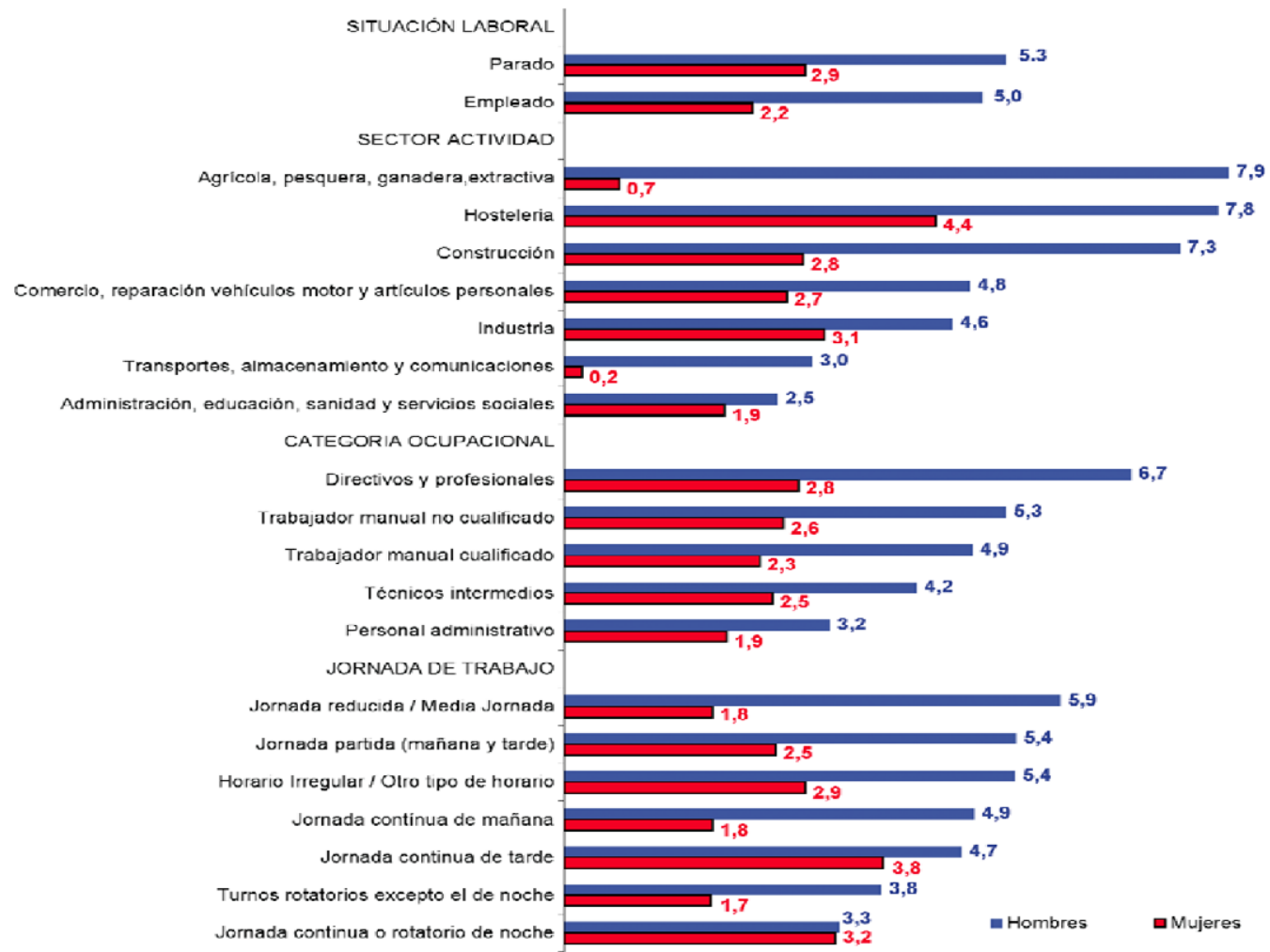

Fuente: Encuesta Domiciliaria sobre Alcohol y Drogas en España. EDADES-2007-08

Observatorio Español sobre Drogas. 


\section{ANEXO 4. CUESTIONARIO MALT}

Detección del alcoholismo

\begin{tabular}{lll}
\hline \multicolumn{1}{c}{ MALT-O. A rellenar por el médico } & SI & NO \\
\hline 1. & Enfermedad hepática \\
2. & Polineuropatía \\
3. & Delirium tremens \\
4. & Consumo alcohólico superior a los $150 \mathrm{ml}$ diarios en los últimos meses. \\
5. & Consumo alcohólico superior a los $300 \mathrm{ml}$, al menos una vez al mes. \\
6. Hedor alcohólico & \\
7. & Los familiares o allegados ya han buscado, en una ocasión, consejo acerca del problema \\
& alcohólico del paciente
\end{tabular}

Cuestionario MALT S. A rellenar por el propio paciente

1. En los últimos tiempos me tiemblan a menudo las manos

2. A temporadas, sobre todo por la mañana, tengo una sensación

3. Alguna vez he intentado calmar la resaca, el temblor o la náusea

4. En la actualidad, me siento amargado por mis problemas y dificultades

5. No es raro que beba alcohol antes del desayuno o el almuerzo

6. Tras los primeros vasos de una bebida alcohólica, a veces, siento la necesidad irresistible de seguir bebiendo

7. A menudo pienso en el alcohol

8. A veces he bebido alcohol, incluso cuando el médico me lo había prohibido

9. En las temporadas en que bebo más, como menos

10. En el trabajo, me han llamado ya la atención por mis ingestas de alcohol y/o alguna vez he faltado al trabajo por haber bebido

11. Últimamente prefiero beber el alcohol a solas y sin que me vean

11 bis. Bebo de un trago y más de prisa que los demás

12. Desde que bebo más, soy menos activo/a.

13. A menudo me remuerde la conciencia o tengo sentimiento de culpa, después de haber bebido

14. He ensayado un sistema para beber, p.ej. no beber antes de determinadas horas

15. Creo que debería limitar mis ingestas de alcohol

16. Sin alcohol no tendría tantos problemas

17. Cuando estoy excitado bebo alcohol para calmarme

18. Creo que el alcohol está destruyendo mi vida

19. Tan pronto quiero dejar de beber como cambio de idea y vuelvo a que no

20. Otras personas no pueden comprender por qué bebo

21. Si yo no bebiera, me llevaría mejor pareja

23. Si no bebiera, estaría contento conmigo mismo

24. Repetidamente me han mencionado mi "aliento alcohólico"

25. Aguanto cantidades importantes de alcohol sin apenas notarlo

26. A veces, al despertar después de un día de haber bebido mucho, aunque sin embriagarme, no recuerdo en absoluto las cosas que pasaron en la víspera

\section{Valoración}

4 puntos por cada respuesta positiva del MALT O

1 punto por cada respuesta positiva del MALT $S$

\section{Orientación diagnóstica}

0-5 puntos: ausencia de alcoholismo

6-10 puntos: sospecha de alcoholismo o riesgo de alcoholismo

11 o más puntos: alcoholismo 


\section{ANEXO 5. CUESTIONARIO AUDIT}

\section{Cuestionario AUDIT (Babor, De la Fuente, Saunders y Grant, 1989)}

1. ¿Con qué frecuencia consume alguna bebida alcohólica?
(0) Nunca
(1) Una vez al mes
(2) 2 a 4 veces al
(3) 2 a 3 veces a
(4) 4 o más veces
o menos
mes
la semana
a la semana

2. ¿Cuantas bebidas alcohólicas consume en un día cualquiera que está tomando alcohol?
(0) 1 ó 2
(1) 3 ó 4
(2) 5 ó 6
(3) 7 a 9
(3) 10 ó más

3. ¿ Con qué frecuencia consume 6 copas o más en una ocasión?
(0) Nunca
(1) Menos de una
(2) Una vez al mes
(3) Una vez por semana
(4) Diariamente o casi diariamente

4. ¿Cuántas veces, durante el último año, observó que no era capaz de dejar de beber una vez que había empezado?
(0) Nunca
(1) Menos de una vez al mes
(2) Una vez al mes
(3) Una vez por semana
(4) Diariamente o casi diariamente

5. ¿Cuántas veces, durante el último año, ha dejado de cumplir con sus obligaciones a causa del alcohol?
(0) Nunca
(1) Menos de una vez al mes
(2) Una vez al mes
(3) Una vez por semana
(4) Diariamente o casi diariamente

6. ¿En cuántas ocasiones, durante el último año, ha necesitado tomar una copa por la mañana para poder "arrancar", después de haber consumido gran cantidad de alcohol el día anterior?
(0) Nunca
(1) Menos de una vez al mes
(2) Una vez al mes
(3) Una vez por semana
(4) Diariamente o casi diariamente

7. ¿Cuántas veces, durante el último año, se ha sentido culpable, o ha tenido remordimientos después de haber bebido?
(0) Nunca
(1) Menos de una vez al mes
(2) Una vez al mes semana
(3) Una vez por
(4) Diariamente o casi diariamente

8. ¿Con cuánta frecuencia durante el último año ha sido incapaz de recordar lo que había pasado la noche anterior porque había estado bebiendo?
(0) Nunca
(1) Menos de una vez al mes
(2) Una vez al mes
(3) Una vez por semana
(4) Diariamente o casi diariamente

9. ¿Ha tenido algún accidente o ha herido a alguien como consecuencia del consumo de alcohol?
(0) No
(1) Sí, pero no durante el
(2) Sí, durante el último año ultimo año

10. ¿Se ha preocupado algún amigo, familiar, médico u otro profesional sobre su consumo de bebidas alcohólicas, o le han que no beba tanto?
(0) No
(1) Sí, pero no durante el
(2) Sí, durante el último año 


\section{ANEXO 6. ESPECIFICIDAD Y SENSIBILIDAD DE LOS CUESTIONARIOS AUDIT Y CAGE}

\begin{tabular}{lll}
\hline & AUDIT & CAGE \\
\hline SENSIBILIDAD & $56-59 \%$ & $85 \%$ \\
ESPECIFICIDAD & $91-96 \%$ & $90 \%$ \\
\hline
\end{tabular}

Fuente: Campos Arias A, y col Propiedades psicométricas del cuestionario CAGE para consumo abusivo de alcohol: Resultado de tres análisis. Revista Col de Psiquiatría Abril- Junio 2009 Vol 38, Núm 2.

Rosón Hernández B. Consumo de riesgo y perjudicial de alcohol. Prevalecida y métodos de detección en la practica clínica. Revista Galicia Clin 2008; 69 (1): 29-44.

|||||||||||||||||||||||||||||||||||||||||||||||||||||||||||||||||||||||||||||||||||||||||||||||||||||||||||||||||||||||||||||||||||||||||||||||||||||||||||||||||||||||||||||||||||||||||||||||||||||||||||||||||| 